\title{
Flory Enthalpy Parameter at Infinite Dilution of Polymer Solutions Determined by Various Methods
}

\author{
Kenji KAMIDE, Shigenobu MATSudA, and Masatoshi SAITo \\ Fundamental Research Laboratory of Fibers and Fiber-Forming Polymers, \\ Asahi Chemical Industry Co., Ltd., \\ Hacchonawate 11-7, Takatsuki, Osaka 569, Japan
}

(Received July 16, 1987)

\begin{abstract}
The Flory enthalpy parameter at infinite dilution $\kappa_{0}$ was evaluated for atactic polystyrene (PS)-cyclohexane (CH) and-trans-decalin (D) systems by applying the following four methods from literature data: (1) Temperature dependence of vapor pressure and membrane osmotic pressure, (2) critical solution temperature $T_{\mathrm{c}}$ and critical solution concentration $v_{\mathrm{p}}^{\mathrm{c}}$ for a series of solutions of polymers, (3) temperature dependence of the second virial coefficient in the vicinity of the Flory theta temperature $\theta$, and (4) calorimetry. In method 2, Shultz-Flory, Stockmayer, Koningsveld et al. (KKS), and Kamide-Matsuda (KM)'s procedures were applied to estimate $\theta$ and the entropy parameter $\psi_{0}$, from which $\kappa_{0}$ was calculated as $\psi_{0} \theta / T(T$, temperature). $\kappa_{0}$ values at $\theta$ deduced by these methods (in method (2), by KKS and KM treatments) yielded a single master curve against the weight- or number-average molecular weight $\bar{M}_{w}$ or $\bar{M}_{n}$; $\kappa_{0}$ (at $\theta)=0.924 \bar{M}_{w}{ }^{-0.089}$ for PS-CH system. Excellent agreement $( \pm 0.02)$ was confirmed between $\kappa_{0}$ values at $\theta$ deduced by various methods when $\bar{M}_{w}\left(\right.$ or $\bar{M}_{n}$ ) of the polymer was the same. This fact strongly supports the validity of the modified Flory-Huggins solution theory, in which the polymer-solvent interaction parameter $\chi$ depends on the polymer concentration and its molecular weight.
\end{abstract}

KEY WORDS Flory Enthalpy Parameter / Polymer Solution / Polystyrene / Chemical Potential / Osmotic Pressure / Light Scattering / Second Virial Coefficient / Critical Solution Point / Calorimetry /

The pair interaction Flory enthalpy parameter $\kappa$ is defined by the van Laar-Scatchard type relation ${ }^{1}$ :

$$
\kappa=\Delta H_{0} /\left(R T v_{\mathrm{p}}{ }^{2}\right)
$$

where $\Delta H_{0}$ is the partial molar heat of dilution with respect to the solvent, $R$, the gas constant, $T$, Kelvin temperature, $v_{\mathrm{p}}$, the polymer volume fraction.

Calorimetry allows direct determination of $\Delta H_{0}$, and thus $\kappa$. The calorimetric experiments, made by Fujishiro and his students, indicated $\kappa$ not to be constant independent of $T$ and $v_{\mathrm{p}}$, which was first assumed in the original Flory-Huggins theory, but a purely phenomenological parameter, which depends on both $T$ and $v_{\mathrm{p}}{ }^{2}$.

$$
\kappa=\kappa_{0}+\kappa_{1} v_{\mathrm{p}}+\kappa_{2} v_{\mathrm{p}}^{2}+\cdots
$$

$\kappa_{0}$ in eq 2 is given by

$$
\kappa_{0}=\lim _{v_{\mathbf{p}} \rightarrow 0}\left\{\Delta H_{0} /\left(R T v_{\mathbf{p}}^{2}\right)\right\}
$$

Theoretically, $\kappa$ or simply $\kappa_{0}$ can be evaluated by various methods, directly or indirectly as will be described later. In other words, if good agreement between $\kappa$ or $\kappa_{0}$ values evaluated by various methods is confirmed, the theory, on which the principles of the methods are based, is considered thoroughly acceptable to explain all the thermodvnamic properties of polymer solutions.

In 1954, Krigbaum ${ }^{3}$ found that the values of the entropy parameter $\psi_{0}$ (see eq 7) (thus, the 
enthalpy parameter $\kappa_{0}$ at theta temperature (see eq 6)) for atactic polystyrene (PS)cyclohexane $(\mathrm{CH})$ system deduced from the critical solution points ${ }^{4}$ (by his expression, the precipitation temperature) by Shultz-Flory method (eq 8) and from intrinsic viscosity differ from those obtained from the osmotic second virial coefficient $A_{2}$ by factors of about 3 and $1 / 3$, respectively. He concluded that the magnitude of the important entropy parameter $\psi_{0}$ remains in doubt pending on further refinement of existing theories. Thereafter (in 1955), Krigbaum and Carpenter ${ }^{6}$ attempted to settle this problem by estimating $\psi_{0}$ from the temperature dependence of the light scattering second virial coefficient but without success because they obtained for the same polymersolvent system a surprisingly low $\psi_{0}$, which was considered mainly due to large experimental error inherent in the light scattering measurements.

Up to now, as far as authors know, no further attempt to compare $\psi_{0}$ or $\kappa_{0}$ values estimated by various methods has been made. Note that for this purpose $\kappa_{0}$ is preferable to $\psi_{0}$, because the former can be determined directly without using any particular theory of a polymer solution.

We believe that for the past 30 years the situation has drammatically changed and a great deal of progress has been made and among the latest developments are the follows:

(1) the reliability of $A_{2}$ determination by the light scattering (LS) method was improved remarkably as a result of well-established experimental techniques and instrumental advance (for example by using laser light) and a sufficient accumulation of the experimental data with high degree of precision for monodisperse polymer solutions has been made very systematically,

(2) prominent progress was achieved in theory on the critical phenomena of polymer solutions,

(3) advance of calorimetry allowed us to apply it to the polymer solutions, which have very small partial heats of dilution and have relatively larger viscosities,

(4) it was confirmed that any thermodynamic parameter can not be accurately determined from hydrodynamic properties including the limiting viscosity number for lack of sound theoretical background.

In this article we calculated $\kappa_{0}$ for atactic PS-CH and trans-decalin (D) systems by applying various direct or indirect methods to literature data available in order to clarify whether $\kappa_{0}$ values thus evaluated coincide with each other.

\section{THEORETICAL BACKGROUND}

$\kappa_{0}$ can be determined by the following methods:

(1) The temperature dependence of vapor pressure and osmotic pressure through use of the relations ${ }^{1}$

$$
\kappa_{0}=\lim _{v_{\mathfrak{p}} \rightarrow 0}\left\{1 /\left(R T v_{\mathbf{p}}^{2}\right)\right\}\left\{\partial\left(\Delta \mu_{0} / T\right) / \partial(1 / T)\right\}_{\mathbf{P}, v_{\mathbf{p}}}
$$

and

$$
\Delta \mu_{0}=R T \ln \left(P_{0} / P_{0}{ }^{0}\right)=-\pi / V_{0}{ }^{0}
$$

Here, $\Delta \mu_{0}$ is the chemical potential of the solvent in solution, $P_{0}$, the vapor pressure of the solvent component in solution, $P_{0}{ }^{0}$, the vapor pressure of the pure solvent, $\pi$, the osmotic pressure, $V_{0}^{0}$, the molar volume of the solvent. The partial differentiation of $\Delta \mu_{0} / T$ with respect to $1 / T$ is carried out under constant pressure and constant composition except the polymer.

(2) The critical phenomena (critical solution temperature $T_{\mathrm{c}}$ and critical polymer concentration $\left.v_{\mathbf{p}}{ }^{\mathrm{c}}\right) . \kappa_{0}$ are related to Flory theta temperature $\theta$ and Flory entropy parameter $\psi_{0}$ through the definition of $\theta{ }^{1}$

$$
\kappa_{0}=\theta \psi_{0} / T
$$


with

$$
\begin{aligned}
\psi_{0} & =\lim _{v_{\mathbf{p}} 0 \rightarrow 0}\left(\Delta S_{0}-\Delta S_{0}{ }^{\text {comb }}\right) /\left(R v_{\mathbf{p}}{ }^{2}\right) \\
& =\lim _{v_{\mathbf{p}} \rightarrow \rightarrow 0}\left(\Delta \mu_{0}-\Delta H_{0}-T \Delta S_{0}{ }^{\text {comb }}\right) /\left(R T v_{\mathbf{p}}{ }^{2}\right)
\end{aligned}
$$

$\Delta S_{0}$ is the partial molar entropy of dilution and $\Delta S_{0}{ }^{\text {comb }}$, the combinatorial entropy term.

$\theta$ and $\psi_{0}$ can be evaluated from $T_{\mathrm{c}}$ (and $v_{\mathrm{p}}^{\mathrm{c}}$ ) for a series of solutions of polymers having different molecular weights by the following four methods:

\section{(a) Shultz and Flory (SF) Method M $^{4}$}

Shultz and Flory derived a relation between $T_{\mathrm{c}}$ and the weight-average molar volume ratio of the polymer to the solvent $X_{w}$ given by

$$
1 / T_{\mathrm{c}}=\left(1 / \theta \psi_{0}\right)\left(1 / X_{w}{ }^{1 / 2}+1 /\left(2 X_{w}\right)\right)+1 / \theta
$$

The slope of the plot of $1 / T_{c}$ against $\left(1 / X_{w}{ }^{1 / 2}\right.$ $\left.+1 /\left(2 X_{w}\right)\right)$ gives $1 / \theta \psi_{0}$ and its intercept gives $1 / \theta$. It must be noticed that eq 8 is strictly valid only for a monodisperse polymer-single solvent system. Furthermore, the $\chi$-parameter is given in a well-known relation

$$
\begin{aligned}
\Delta \mu_{0}= & R T\left\{\ln \left(1-v_{\mathrm{p}}\right)\right. \\
& \left.+\left(1-1 / X_{n}\right) v_{\mathbf{p}}+\chi v_{\mathbf{p}}{ }^{2}\right\}
\end{aligned}
$$

( $X_{n}$ is the number-average molar volume ratio of the polymer to the solvent) and is assumed to be independent of the polymer molecular weight and the polymer concentration.

\section{(b) Stockmayer Method ${ }^{7}$}

Stockmayer derived a relation (eq 10) for multi-component polymers-single solvent system, in which the $\chi$ parameter is independent of $v_{p}$ and molar volume ratio of the polymer to the solvent $X$.

$$
\begin{aligned}
1 / T_{\mathrm{c}}= & \left(1 /\left(2 \theta \psi_{0}\right)\right)\left\{\left(1 / X_{w}{ }^{1 / 2}+\left(X_{z} / X_{w}\right)^{1 / 2}\right)\right. \\
& \left.\times\left(1 / X_{w}{ }^{1 / 2}+\left(X_{w} / X_{z}\right)^{1 / 2}\right)\right\} \\
& +\left(1-1 /\left(2 \psi_{0}\right)\right) / \theta
\end{aligned}
$$

Here $X_{z}$ is the $z$-average $X$. Plot of $1 / T_{\mathrm{c}}$ as a function of $\left\{\left(1 / X_{w}{ }^{1 / 2}+\left(X_{z} / X_{w}\right)^{1 / 2}\right)\left(1 / X_{w}{ }^{1 / 2}\right.\right.$ $\left.\left.+\left(X_{w} / X_{z}\right)^{1 / 2}\right)\right\} / 2$ yields $\left(1 /\left(\theta \psi_{0}\right)\right)$ from slope and $\left(1-1 /\left(2 \psi_{0}\right)\right) / \theta$ as an intercept at

$$
\begin{aligned}
& \left\{( 1 / X _ { w } { } ^ { 1 / 2 } + ( X _ { z } / X _ { w } ) ^ { 1 / 2 } ) \left(1 / X_{w}{ }^{1 / 2}+\right.\right. \\
& \left.\left.\left(X_{w} / X_{z}\right)^{1 / 2}\right)\right\} / 2=1 / 2 .
\end{aligned}
$$

Therefore, $\theta$ and $\psi_{0}$ can be unambiguously determined.

\section{(c) Koningsveld et al. (KKS) Method ${ }^{8}$}

An alternative expression of neutral equilibrium conditions is given by

$$
\begin{aligned}
Y & \equiv g_{1}-g_{2}+4 g_{2} v_{\mathrm{p}}^{\mathrm{c}} \\
& =\left\{1 /\left(1-v_{\mathrm{p}}^{\mathrm{c}}\right)^{2}-X_{z} /\left(X_{w} v_{\mathrm{p}}\right)^{\mathrm{c}}\right\} / 6
\end{aligned}
$$

with

$$
\begin{aligned}
g & =g_{0}+g_{1} v_{\mathbf{p}}+g_{2} v_{\mathrm{p}}{ }^{2} \\
& =\left(1 / v_{0}\right) \int_{1-v_{0}}^{1} \chi \mathrm{d}\left(1-v_{0}\right)
\end{aligned}
$$

where $g$ is a thermodynamic interaction parameter, related to $\chi$-parameter through eq $12-\mathrm{b}, v_{0}$, volume fraction of the solvent $\left(=1-v_{\mathrm{p}}\right) \cdot g_{1}$ and $g_{2}$ in eq 11 are calculated from $v_{\mathrm{p}}^{\mathrm{c}}, X_{w}$, and $X_{z}$ by applying the curve fitting method to eq. 11 .

$g_{0}$, defined in eq 12-a, is evaluated from $g_{1}$ and $g_{2}$, and $v_{\mathrm{p}}^{\mathrm{c}}$ and $X_{w}$, using the spinodal equation,

$$
\begin{aligned}
2 g_{0} \equiv 2 & \left(g_{00}+g_{01} / T_{\mathrm{c}}\right) \\
= & 1 /\left(1-v_{\mathrm{p}}^{\mathrm{c}}\right)+1 /\left(v_{\mathrm{p}}^{\mathrm{c}} X_{w}\right) \\
& +2 g_{1}\left(1-3 v_{\mathrm{p}}^{\mathrm{c}}\right)+6 g_{2}\left(1-2 v_{\mathrm{p}}^{\mathrm{c}}\right) v_{\mathrm{p}}^{\mathrm{c}}
\end{aligned}
$$

Plot of $g_{0}$, obtained thus, versus $T_{0}{ }^{-1}$ enables us to estimate $g_{00}$ and $g_{01}$. Considering the relation

$$
\chi_{0} \equiv \lim _{v_{\mathbf{p}} \rightarrow 0} \chi=g_{00}-g_{1}+g_{01} / T
$$

we obtain the following relations:

$$
\begin{gathered}
\psi_{0}=1 / 2-g_{00}+g_{01} \\
\theta=g_{01} / \psi_{0}
\end{gathered}
$$




$$
\kappa_{0}=g_{01} / T
$$

Then, $\kappa_{0}$ can be determined from eq 17 .

\section{(d) Kamide-Matsuda Method ${ }^{9}$}

At the critical point, the following equations can be derived, ${ }^{9}$

$$
\begin{aligned}
& 1 /\left(X_{w} v_{\mathrm{p}}^{\mathrm{c}}\right)+1 /\left(1-v_{\mathrm{p}}^{\mathrm{c}}\right) \\
& -\chi_{0}^{\mathrm{c}}\left(2+\sum_{j=1} p_{j}(j+2) v_{\mathrm{p}}^{\mathrm{c} j}\right)=0
\end{aligned}
$$

and

$$
\begin{aligned}
& 1 /\left(1-v_{\mathrm{p}}^{\mathrm{c}}\right)^{2}-X_{z} /\left(X_{w} v_{\mathrm{p}}^{\mathrm{c}}\right)^{2} \\
& -\chi_{0}^{\mathrm{c}}\left(\sum_{j=1} p_{j} j(j+2) v_{\mathrm{p}}^{\mathrm{c} j-1}\right)=0
\end{aligned}
$$

where $p_{j}$ is the concentration dependence coefficient given in eq 20 ,

$$
\chi=\chi_{0}\left(1+\sum_{j=1} p_{j} v_{\mathrm{p}}^{j}\right)
$$

Here $\chi_{0}$ is the concentration independent coefficient and $\chi_{0}{ }^{\mathrm{c}}$ is the critical $\chi_{0}$.

$\chi_{0}{ }^{\mathrm{c}}$ is related to $T_{\mathrm{c}}, \psi_{0}$, and $\theta$ through the relation

$$
1 / T_{\mathrm{c}}=\chi_{0}{ }^{\mathrm{c}} /\left(\theta \psi_{0}\right)+(1 / \theta)\left(1-1 /\left(2 \psi_{0}\right)\right)
$$

First, calculate $\chi_{0}{ }^{\mathrm{c}}$ and $v_{\mathrm{p}}{ }^{\mathrm{c}}$ (theo) by solving eq 18 and 19 with assumed values of $p_{1}$ and $p_{2}$. Next determine the most probable $p_{1}$ and $p_{2}$ as a pair which gives a minimum value of the square average of the difference between $v_{\mathrm{p}}{ }^{\mathrm{c}}$ (theo) and $v_{\mathrm{p}}{ }^{\mathrm{c}}$ (exp). Substituting $\chi_{0}{ }^{\mathrm{c}}$, calculated for the most probable $p_{1}$ and $p_{2}$ and the experimental critical temperature $T_{\mathrm{c}}(\exp )$ into eq 21 , and plotting the relation of $1 / T_{c}(\exp ) v s$. $\chi_{0}{ }^{\mathrm{c}}$ (theo), we obtain $\theta$ and $\psi_{0}$.

In the former two methods only $T_{\mathrm{c}}$ is necessary and in the latter two methods $v_{\mathrm{p}}{ }^{\mathrm{c}}$ together with $T_{\mathrm{c}}$ are needed for analysis and $p_{1}$ and $p_{2}$ are obtained simultaneously $\left(g_{1}\right.$ and $g_{2}$, obtained by KKS method, are readily transformed to $p_{1}$ and $\left.p_{2}{ }^{9}\right)$. These four methods presume that $\chi_{0}{ }^{\mathrm{c}}$ is independent of $X$.
(3) Temperature dependence of $A_{2}$ by membrane osmometry or light scattering measured in the vicinity of $\theta$ temperature (eq 22) via $\theta$ and $\psi_{0}{ }^{2}$

$$
\psi_{0}=\left(V_{0}^{0} / \bar{v}^{2}\right) \theta\left(\partial A_{2} / \partial T\right)_{\theta}
$$

Here $\bar{v}$ is the specific volume of polymer and note that $\psi_{0}$ estimated by eq 22 corresponds to the finite molecular weight. $\theta$ is determined as the temperature at which $A_{2}$ becomes zero. $\kappa_{0}$ can be evaluated by putting $\theta$ and $\psi_{0}$ into eq 6 .

\section{(4) Calorimetry}

The heat of dilution $\Delta H$ and accordingly $\Delta H_{0}\left(\equiv\left(\partial \Delta H / \partial n_{0}\right)_{\mathrm{T}, \mathrm{P}}, n_{0}\right.$ is the mole number of the solvent) can be directly measured with calorimetry.

Figure 1 demonstrates schematic routes of determination for $\kappa_{0}$.

\section{APPLICATION TO EXPERIMENTAL DATA}

The thermodynamic properties of atactic PS-CH system have hitherto been most thoroughly investigated and the literature data available for this system were chosen for estimating $\kappa_{0}$. As a supplement the literature data for atactic PS-trans-decalin system were also analyzed.

Krigbaum and Geymer ${ }^{10}$ estimated the partial molar heat of dilution through use of eq 4 and 5 from the temperature dependence of the osmotic pressure and vapor pressure for a PS sample (the number-average molecular weight $\bar{M}_{n}=4.4 \times 10^{5}$ ) in $\mathrm{CH}$ at $307.2 \mathrm{~K}$ (Table V of ref 10). From their data we obtain $\kappa_{0}=0.21$ for $\bar{M}_{n}=4.4 \times 10^{5}$ as shown in Table I. It is noticed that the measurements were made over the temperature range of $34-44^{\circ} \mathrm{C}$.

Scholte ${ }^{11}$ determined the chemical potential of an atactic monodisperse PS $\left(\bar{M}_{\mathrm{n}}=1.54 \times\right.$ $\left.10^{5}\right)-\mathrm{CH}$ system at $303.2,318.2$, and $338.2 \mathrm{~K}$ from sedimentation equilibrium in the ultracentrifuge. Using the data in Table $\mathrm{V}$ of ref 11 we estimated $\kappa_{0}$ as a function of temper- 
ature and $\kappa_{0}$ was found to be 0.32 at $307.2 \mathrm{~K}$ ( $\simeq \theta$ temperature). Scholte discussed the accuracy of determined parameters including $\Delta \mu_{0}$, concluding that the error in $\Delta \mu_{0}$ could be estimated to between $5 \%$ at low $v_{\mathrm{p}}$ and $10 \%$ at high $v_{\mathrm{p}}$. Then, it is conceivable that $\kappa_{0}$ estimated from Scholte's data is more reliable than that from Krigbaum and Geymer. ${ }^{10}$

Koningsveld et al. ${ }^{8}$ measured the upper critical point $\left(v_{\mathrm{p}}^{\mathrm{c}}\right.$ and $\left.T_{\mathrm{c}}\right)$ for 6 monodisperse PS samples (the weight-average molecular weight $\left.\bar{M}_{w}=3.54-150.0 \times 10^{4}\right)$ in $\mathrm{CH}$ by the phase volume ratio method (see Table I of ref 8 ) and Kuwahara and his coworkers ${ }^{12}$ estimated $v_{\mathrm{p}}{ }^{\mathrm{c}}$ and $T_{\mathrm{c}}$ of the upper critical points for 4 monodisperse PS samples $\left(\bar{M}_{w^{\prime}}=20.0\right.$ $\left.68.0 \times 10^{4}\right)$ and a mixture sample in $\mathrm{CH}$ by the diameter method (see, Table I of ref 12). Saeki et $a{ }^{13}$ determined upper cloud point curves for 6 monodisperse PS samples $\left(\bar{M}_{x^{\prime}}=3.7-\right.$ $270.0 \times 10^{4}$ ) in $\mathrm{CH}$. We estimated the critical solution points as the maximum points of their cloud point curves (see, Figure 1 of ref 13). All these three sets of data (in total 16 samples) were, on the whole, analyzed ac- cording to the methods of SF, Stockmayer, KKS, and KM to estimate $\theta$ and $\psi_{0}$ (accordingly, $\left.\kappa_{0}\right)$. The plots of three sets of data according to each treatment yielded a single common straight line (for example. see Figure

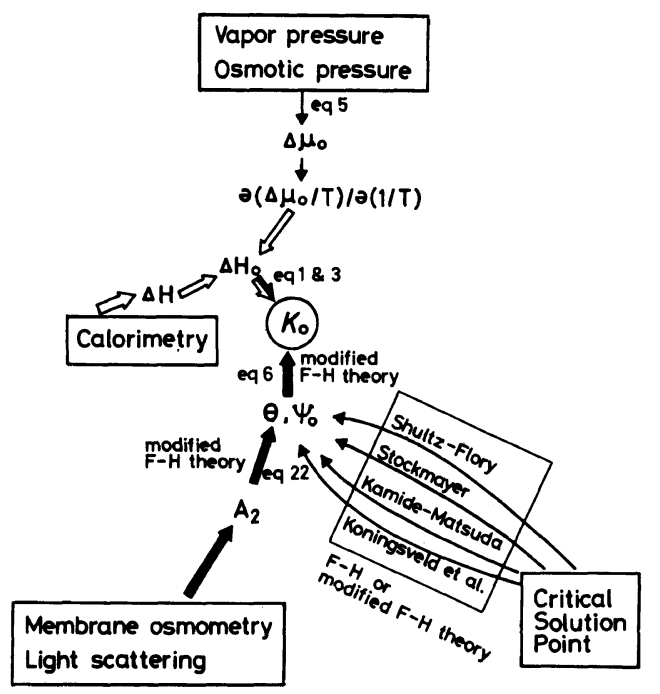

Figure 1. Routes of calculation of the Flory enthalpy parameter from experimental data of vapor pressure, osmotic pressure, light scattering, and critical solution points.

Table I. Flory $\theta$ temperature and entropy parameter $\psi_{0}$ at infinite dilution of the upper critical solution point of atactic polystyrene-cyclohexane system

\begin{tabular}{|c|c|c|c|c|c|}
\hline Method & & $\underline{\theta}$ & $\begin{array}{c}\kappa_{0}\left(\bar{M}_{n} \text { or }\right. \\
\left.\bar{M}_{w} \times 10^{-4}\right)\end{array}$ & & (Data) \\
\hline \multirow[t]{2}{*}{ (1) Chemical potential } & Membrane osmometry & - & $0.21(44)$ & & $(\text { Krigbaum-Geymer })^{10}$ \\
\hline & Sedimentation equilibrium & - & $0.32(15.4)$ & & (Scholte) $^{11}$ \\
\hline \multirow[t]{4}{*}{ (2) Critical point } & Shultz-Flory & 306.2 & $0.75(-)$ & & \\
\hline & Stockmayer & 306.5 & $0.80(-)$ & & (Konningsveld et al., \\
\hline & Konningsveld et al. & 305.2 & $0.29(-)$ ? & {$[0.28]^{\mathrm{a}}$} & Kuwahara et al., \\
\hline & Kamide-Matsuda & 305.1 & $0.27(-)\}$ & $0.26(\infty)$ & Saeki et al. $)^{8,12,13}$ \\
\hline \multirow{8}{*}{$\begin{array}{l}\text { (3) Second virial } \\
\text { coefficient }\end{array}$} & Membrane osmometry & 307.6 & $0.28(\infty)$ & {$[0.28]^{\mathrm{a}}$} & $(\text { Krigbaum })^{3}$ \\
\hline & & 307.6 & $0.27(\infty)\}$ & & $(\text { Krigbaum-Geymer })^{10}$ \\
\hline & Light scattering & 308.0 & $0.19(163)$ & & $(\text { Outer } \text { et al. })^{17}$ \\
\hline & & 308.4 & $0.19(320)$ & & ${\text { (Krigbaum-Carpenter })^{6}}$ \\
\hline & & 307 & $0.39(17)$ & & $(\text { Schulz-Baumann })^{18}$ \\
\hline & & 307.4 & $0.26(30)$ & {$[0.26]^{\mathrm{a}}$} & $(\text { Kotera et al. })^{19}$ \\
\hline & & 308 & $0.22(\infty)$ & & $(\text { Miyaki-Fujita) })^{20,21}$ \\
\hline & & 307.7 & $0.30(\infty)$ & & $(\text { Tong et al. })^{22}$ \\
\hline (4) Heat of dilution & Calorimetry & - & $0.28(\infty)$ & & ${\text { (Fujihara })^{25}}^{25}$ \\
\hline
\end{tabular}

a Averaged value. 
5 of ref 9). In KKS and KM methods, we estimated $X_{z}$ assuming the Schulz-Zimm type molecular weight distribution for all PS samples of Kuwahara et al. ${ }^{12}$ and Saeki et $a l$.'s ${ }^{13}$ papers. The $\theta$ and $\kappa_{0}$ values obtained are summarized in Table I. Application of the KKS method to the above literature data gives $p_{1}=0.623$ and $p_{2}=0.308$ and that of the KM method to the same data yields $p_{1}=$ 0.624 and $p_{2}=0.190$, together with $\theta$ and $\psi_{0}$. These values are in fairly good agreement with those evaluated by osmotic pressure, ${ }^{3,10}$ isothermal distillation, ${ }^{10}$ vapor pressure, ${ }^{10}$ phase separation, ${ }^{14,15}$ cloud point curves, ${ }^{16}$ and ultracentrifuge. ${ }^{11}$ Previously Kamide and Matsuda ${ }^{9}$ pointed out that the ShultzFlory and Stockmayer theories yield incorrectly low $v_{\mathrm{p}}^{\mathrm{c}}$ value: $v_{\mathrm{p}}^{\mathrm{c}}(\exp )>v_{\mathrm{p}}^{\mathrm{c}}$ (theo) and the absolute magnitude of $\psi_{0}$ values estimated by methods of Koningsveld et al. ${ }^{8}$ and Kamide and Matsuda ${ }^{9}$ are only half or $1 / 3$ those by the SF and Stockmayer methods for PS-CH system.

$\kappa_{0}$ was found to be 0.75 by the SF method and 0.80 by Stockmayer. These values are more than three times larger than those by the other methods. Shultz and Flory ${ }^{4}$ determined precipitation temperature (i.e., cloud point curve) of four PS fractions in $\mathrm{CH}$ and obtained $\theta=307.2 \mathrm{~K}$ and $\kappa_{0}=1.055_{8}$ at $\theta$ using their method. They noticed that the parameters deduced from this method appeared to be unacceptable. Saeki et al. ${ }^{13}$ evaluated $\theta$ and $\kappa_{0}$ at $\theta$ to be $486 \mathrm{~K}$ and 0.79 by the SF method. Undoubtedly, SF and Stockmayer methods overestimate $\psi_{0}$ (accordingly, $\kappa_{0}$ ). Therefore, the most probable value of $\kappa_{0}$ at $\theta$, estimated from the critical points is an average $(0.28)$ of those $(0.29$ and 0.27$)$ by Koningsveld et al. method and Kamide-Matsuda method (see, the fifth column of Table I).

For atactic PS-CH system, the membrane osmotic pressure (MO) and light scattering (LS) measurements have been carried out to estimate $A_{2}$ over a temperature range including $\theta$ temperature by Krigbaum (MO), ${ }^{3}$
Krigbaum and Geymer (MO), ${ }^{10}$ Outer et al. (LS), ${ }^{17}$ Krigbaum and Carpenter (LS), ${ }^{6}$ Schulz and Baumann (LS), ${ }^{18}$ Kotera et al. (LS), ${ }^{19}$ Miyaki-Fujita (LS), ${ }^{20,21}$ and Tong et al. (LS). ${ }^{22}$

The results of the present analysis for the literature data are assembled in Table II. Here, we constructed the plots of $A_{2}$ against $T$ using the literature data, from which $\left(\partial A_{2} / \partial T\right)_{\theta}$ was estimated. In the table $\kappa_{0}$ at $308 \mathrm{~K}$ (i.e., the most probable $\theta$ temperature) is demonstrated.

The value of $\theta$ for the samples in Krigbaum's study, except sample code H-2-8, is $305.6 \pm 0.4 \mathrm{~K}$, which is somewhat lower than those estimated by others for this system. Krigbaum $^{3}$ ascribed this disagreement to traces of moisture which entered the polymer solutions in the $313.2 \mathrm{~K}$ bath. The data for sample code H-2-8 were obtained using adequate precautions to prevent contamination of the solutions with water. The effect of water contamination on $\kappa_{0}$ seems insignificant.

Figure 2 shows $\kappa_{0}$ at $\theta$ estimated by the $A_{2}$ method for atactic PS-CH system, as function of $\bar{M}_{w}{ }^{-1}$ or $\bar{M}_{n}{ }^{-1}$. In the figure the $\kappa_{0}$ value (0.32), obtained from the temperature dependence of the chemical potential by Scholte, ${ }^{11}$ is shown for comparison.

Least square straight lines $a$ and $b$ in the figure pass through the observed data points of Krigbaum $^{3}$ (except for sample code H-2-8) and Krigbaum and Geymer, ${ }^{10}$ respectively. From these lines, we can evaluate $\kappa_{0}$ at infinite molecular weight to be 0.28 from Krigbaum's data and 0.27 from Krigbaum and Geymer's data $(0.28$, on average). These values are also presented in Table I for comparison. The $\kappa_{0}$ values obtained from Krigbaum's and by Krigbaum and Geymer's data have a small but significant tendency of decreasing with an increase in $\bar{M}_{n}$.

It is probably true to say that as early as the mid 1950s the membrane osmometry had been established already as an accurate and reliable method for estimating $A_{2}$, but the light scattering technique remained in 1950 s only in em- 
Table II. Evaluation of Flory $\kappa_{0}$ parameter from the temperature dependence of the second virial coefficient $A_{2}$ by membrane osmometry and light scattering for atactic polystyrene-cyclohexane system

\begin{tabular}{|c|c|c|c|c|c|c|c|}
\hline \multirow{2}{*}{ Method } & \multirow{2}{*}{ Reporter (Year) } & \multirow{2}{*}{$\begin{array}{l}\text { Sample } \\
\text { code }\end{array}$} & $\bar{M}_{w}\left(\bar{M}_{n}\right)$ & Temp range & $\left(\partial A_{2} / \partial T\right)_{\theta}$ & $\theta$ & \multirow{2}{*}{$\begin{array}{cl} & \kappa_{0} \\
\text { at } & 308 \mathrm{~K}\end{array}$} \\
\hline & & & $\times 10^{-4}$ & $\mathrm{~K}$ & $\times 10^{6} \mathrm{~cm}^{3} \mathrm{molg}^{-1} \mathrm{~K}$ & $\mathrm{~K}$ & \\
\hline \multirow{7}{*}{$\begin{array}{l}\text { Membrane } \\
\text { osmometry }\end{array}$} & \multirow[t]{5}{*}{ Krigbaum (1954) } & L5-6 & $(5.05)$ & & 9.53 & 305.8 & 0.37 \\
\hline & & HA-9 & $(12.5)$ & & 8.72 & 305.8 & 0.34 \\
\hline & & M2-2 & (35.9) & $303-323$ & 7.84 & 305.6 & 0.30 \\
\hline & & H1-4 & $(56.6)$ & & 7.22 & 305.4 & 0.28 \\
\hline & & $\mathrm{H} 2-8$ & $(20.3)$ & & 9.14 & 307.6 & 0.36 \\
\hline & \multirow{2}{*}{$\begin{array}{l}\text { Krigbaum-Geymer } \\
\text { (1959) }\end{array}$} & F-I & $(7.2)$ & 313,322 & 8.9 & 307.2 & 0.35 \\
\hline & & F-II & $(44.0)$ & $303,313,323$ & 7.2 & 307.9 & 0.28 \\
\hline \multirow[t]{19}{*}{$\begin{array}{l}\text { Light } \\
\text { scattering }\end{array}$} & $\begin{array}{l}\text { Outer } \text { et al. } \\
\quad(1950)\end{array}$ & B-2 & 163 & $300-314.5$ & 4.25 & 308 & 0.17 \\
\hline & $\begin{array}{l}\text { Krigbaum- } \\
\quad \text { Carpenter (1955) }\end{array}$ & HB2-3 & & 320 & 4.76 & 308.4 & 0.19 \\
\hline & $\begin{array}{l}\text { Schulz-Baumann } \\
\quad(1961)\end{array}$ & - & 17 & - & 10 & 307 & 0.39 \\
\hline & $\begin{array}{l}\text { Kotera-Saito- } \\
\text { Fujisaki (1963) }\end{array}$ & SMI & $30(15)$ & $303.2-323.2$ & 6.76 & 307.4 & 0.26 \\
\hline & \multirow{10}{*}{$\begin{array}{l}\text { Miyaki-Fujita } \\
\quad(1981)\end{array}$} & IK $1500-1$ & 5680 & $306-323$ & 4.62 & 307.9 & 0.18 \\
\hline & & IK $1500-2$ & 3900 & $306-323$ & $j .34$ & 307.9 & 0.21 \\
\hline & & BK2500-1 & 3190 & $306-328$ & 5.86 & 307.8 & 0.23 \\
\hline & & BK 2500-2 & 2320 & $306-323$ & 5.95 & 307.8 & 0.23 \\
\hline & & BK2500-3 & 1500 & $305-328$ & 6.62 & 308.3 & 0.26 \\
\hline & & BK2500-4 & 876 & $305-328$ & 5.58 & 308.3 & 0.22 \\
\hline & & $\mathrm{Cl}$ & 647 & 304,328 & 6.01 & 308.2 & 0.24 \\
\hline & & $\mathrm{F} 450$ & 480 & $303-328$ & 5.43 & 308.9 & 0.21 \\
\hline & & F288 & 308 & $305-328$ & 5.91 & 307.3 & 0.23 \\
\hline & & F126 & 134 & $302-328$ & 7.04 & 307.3 & 0.27 \\
\hline & \multirow[t]{5}{*}{ Tong et al. (1984) } & $\mathrm{F} 1$ & 1.00 & $307.7 \mathrm{~K} \sim$ & & & \\
\hline & & $\mathrm{F} 2$ & 2.15 & a value & & & \\
\hline & & F4 & 4.36 & about $1 \mathrm{~K}$ & 8.4 & 307.7 & 0.30 \\
\hline & & F20 & 18.1 & below the & & & \\
\hline & & F40 & 49.8 & cloud point & & & \\
\hline
\end{tabular}

bryo, being not as precise as the osmotic pressure as Krigbaum and Carpenter noticed. ${ }^{6}$ Therefore, the literature LS data at that time may contain large experimental uncertainty. However, an inspection of Figure 2 leads us to the conclusion that $\kappa_{0}$ values estimated from the classical LS $A_{2}$ data by Outer et al., Krigbaum-Carpenter, Schulz-Baumann, and Kotera et al. decrease rapidly with an increase in $\bar{M}_{w}$ and for PS samples with $\bar{M}_{w}>10^{6} \kappa_{0}$ was found to be below 0.20 , which is significantly lower than those estimated by $\mathrm{MO}$ $\left(\bar{M}_{n}=5.50-56.6 \times 10^{4}\right)$. All these classical LS data except for that by Outer et al. do not deviate strongly from a common relation obtained by classical MO and recent LS measurements.

Miyaki and Fujita ${ }^{20,21}$ carried out a tremendously elaborate work in measuring $A_{2}$ for ten very high molecular weight atactic PS samples $\left(\bar{M}_{w}=1.34 \times 10^{6}-5.68 \times 10^{7}\right)$ in $\mathrm{CH}$ at three to seven different temperatures ranging 309.2 to $328.2 \mathrm{~K}$. Except for a fraction (sample code IK $\left.1500-1, \bar{M}_{u^{\prime}}=5.68 \times 10^{6}\right)$, almost the constant value of $\kappa_{0}(=0.23 \pm 0.01$, at $308 \mathrm{~K})$ was obtained over a wide range of $\bar{M}_{w}(8.76 \times$ 


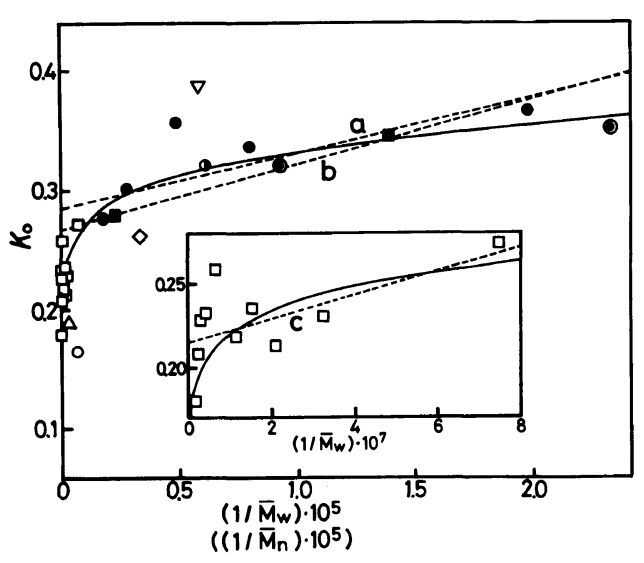

Figure 2. Molecular weight dependence of Flory enthalpy parameter at infinite dilution $\kappa_{0}$, evaluated by the temperature dependence of the chemical potential and of the second virial coefficient $A_{2}$ in vicinity to theta temperature and by calorimetry for atactic polystyrenecyclohexane system: Krigbaum $^{3}$ (membrane osmometry (MO)); $\mathbf{a}$, Krigbaum-Geymer ${ }^{10}$ (MO); O, Scholte $^{11}$ (ultracentrifuge); $\triangle$, Krigbaum-Carpenter ${ }^{6}$ (light scattering (LS)); $\nabla$, Schulz-Baumann ${ }^{18}$ (LS); $\diamond$, Kotera et al. ${ }^{19}$ (LS); $\bigcirc$, Outer et al. ${ }^{17}$ (LS); $\square$, Miyaki ${ }^{21}$ (LS); O, Fujihara ${ }^{25}$ (calorimetry). Solid line, master curve of all data; broken line a, drawn pass through the data points of Krigbaum; b, Krigbaum-Carpenter. Inserted figure, enlarged plots of Miyaki et al. data. Broken line $\mathrm{c}$, drawn pass through the data points of Miyaki et al.

$\left.10^{5}-6.47 \times 10^{6}\right)$ as shown in Table II. The figure inserted in Figure 2 shows the enlarged plots in the lower $\bar{M}_{w}{ }^{-1}$ region. $\kappa_{0}$ values thus determined by Miyaki and Fujita scatter fairly largely, probably due to experimental difficulty of LS measurements of solutions of polymers with $\bar{M}_{w^{\prime}} \sim 10^{7} . \kappa_{0}=0.22$ at $308 \mathrm{~K}$ was obtained for $\bar{M}_{w^{\prime}}=\infty$ from line $\mathrm{c}$ in the figure, constructed by the least-squares method. Tong et $a l .{ }^{22}$ determined $A_{2}$ of five monodisperse PS samples in the temperature range from $307.7 \mathrm{~K}$ to about $1 \mathrm{~K}$ above the cloud point and they found $\mathrm{A}_{2}$ for monodisperse PS in $\mathrm{CH}$ below $\theta$ to be independent of $M$, within the ranges of $T$ and $M$ studied. Since unfortunately the $A_{2}$ values were not described in their paper, we estimated $\left(\partial A_{2} / \partial T\right)_{\theta}$ from the initial slope of the plot of $-A_{2} v s . T^{-1}$ (Figure 4 of ref 22), to be 0.30 .

Table I contains $\theta$ and $\kappa_{0}$ values estimated from the temperature dependence of $A_{2}$. Plural samples were employed in the studies of Krigbaum, ${ }^{3}$ Krigbaum-Geymer, ${ }^{10}$ MiyakiFujita, ${ }^{20,21}$ and Tong et al. ${ }^{22}$ The $\kappa_{0}$ value at infinite molecular weight for each study is shown in the table.

If all the data points in Figure 2 are treated equally, although they scatter markedly, we can conclude that $\kappa_{0}$ decreases slightly with increasing $\bar{M}_{w}$ or $\bar{M}_{n}$ or can be roughly regarded as constant up to $\bar{M}_{w}$ or $\bar{M}_{n}<\sim 1 \times 10^{6}$ and further, the molecular weight dependence of $\kappa_{0}$ tends to become remarkably large for polymer with $\bar{M}_{w}$ or $\bar{M}_{n}>3 \times 10^{7}$. It is particularly noteworthy that $\kappa_{0}$ extrapolated to $\bar{M}_{w}=\infty\left(\kappa_{0}(\infty)\right)$ depends definitely on the molecular weight range, over which the experimental data points were obtained and from which the extrapolation was made.

Amaya and Fujishiro ${ }^{23}$ reported the heat of dilution $\Delta H$, obtained with a calorimetry specially designed and constructed, for five $\mathrm{CH}$ solutions of an atactic PS (unfractionated sample, $X=275$ ) at $303.2 \mathrm{~K}$. From Table I of ref 23 we can calculate $\kappa_{0}$ at $298.2 \mathrm{~K}$ to be 6.0 . This value is unbelievably large. In this case the measurements were carried out almost 4 $5 \mathrm{~K}$ below the $\theta$ temperature and Amaya and Fujishiro ${ }^{23}$ pointed out that it may be reasonable to suppose that in the solution at $303.2 \mathrm{~K}$ there remains more or less aggregations of polymers. Schulz and Horbach ${ }^{24}$ measured $\Delta H$ directly for nine atactic PS fractions (the viscosity-average molecular weight $\bar{M}_{v}=400$ $1.7 \times 10^{4}$ ) in $\mathrm{CH}$ at $296.2 \mathrm{~K}$ and for two PS sample $\left(\bar{M}_{v}=1200\right.$ and $\left.1.1 \times 10^{4}\right)$ they obtained $\Delta H_{0}$ as a function of the polymer concentration $v_{\mathrm{p}}$. $\kappa$, calculated by eq 1 from the experimental $\Delta H_{0}$ (Table 7 of ref 24), indicated a strong negative molecular weight dependence. From these data, however, we cannot evaluate an accurate $\kappa$ or $\kappa_{0}$ at infinite molecular weight in $\theta$ solvent $(\simeq 307.5 \mathrm{~K})$. For example, we obtained $\kappa_{0}=0.9$ for PS with 
$\bar{M}_{v}=1.1 \times 10^{4}$, but this value is almost 2.5 times larger than that $(0.4)$ estimated from Fujihara's data ${ }^{25}$ for monodisperse PS with $\bar{M}_{w}=1.02 \times 10^{4}$ at $307.2 \mathrm{~K}$. The difference in temperature of measurements provides at least a partial explanation of this great difference. From Abb. 4 of ref 24 for atactic PS $\left(\bar{M}_{w^{\prime}}=\right.$ $6.8 \times 10^{4}$ ), in $\mathrm{CH}$ (the polymer concentration $10 \%$ ) (cited from Cantow's data ${ }^{26}$ ) it is shown that the $\kappa$ value at $\theta$ is nearly half that at $297.2 \mathrm{~K}$.

By overcoming numerous experimental difficulties encountered in calorimetry Fujihara ${ }^{25}$ succeeded in measuring directly $\Delta H_{0}$ of four monodisperse PS samples $\left(\bar{M}_{u^{\prime}}=6.2 \times 10^{3}\right.$ $1.07 \times 10^{5}$ ) in $\mathrm{CH}$ at $307.2 \mathrm{~K}$. According to him, the PS-CH system is a large endothermic solution, decreasing $\Delta H_{0}$ with increasing $\bar{M}_{w}$. First we constructed the plot of $\kappa$ against $v_{\mathrm{p}}$ for each sample from the data in Table I of ref 25 and estimated $\kappa_{0}$ as an intercept of the above plot. Figure 3 shows the molecular weight dependence of $\kappa_{0}$ determined thus. $\kappa_{0}$ decreases noticeably with an increase in $\bar{M}_{w}$. The data points except for the highest $\bar{M}_{w}$ sample can be reasonably represented by a straight line, yielding $\kappa_{0}=0.33$ at $\bar{M}_{w}=\infty$. If all the data points are taken into consideration of the extrapolation procedure, we obtain $\kappa_{0}=0.28$ at $\bar{M}_{w}=\infty$.

The molecular weight range covered by calorimetry is somewhat shifted to a side lower than that used for MO and LS experiments, due to ease in conducting the experiments. $\kappa_{0}$ values for the two highest $\bar{M}_{w}$ samples by calorimetry are plotted for comparison in Figure 2. It is noted that the chemical potential method and calorimetry give almost the same $\kappa_{0}$ value with those by $A_{2}$ method for the same $\bar{M}_{w}$, polymer. $\kappa_{0}$ was found by the chemical potential method ${ }^{11}$ for $\bar{M}_{n}=15.4 \times 10^{4}$ to be 0.32 and by calorimetry ${ }^{25}$ for $\bar{M}_{w}=10.7 \times 10^{4}$ to be 0.31 , which should be compared with 0.34 by $A_{2}$ method $\left(\mathrm{Krigbaum}^{3}\right)$ for $\bar{M}_{w}=$ $12.5 \times 10^{4}$ and 0.32 by $A_{2}$ method (Tong et al. ${ }^{22}$ ) for $\bar{M}_{w}=1-18.1 \times 10^{4}$. Tong et al.'s

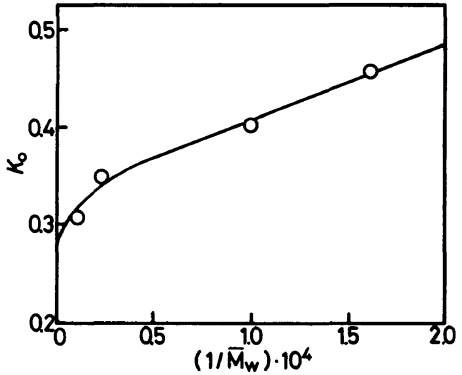

Figure 3. Plots of Flory enthalpy parameter at infinite dilution $\kappa_{0}$, evaluated directly by Fujihara $^{25}$ using calorimetry, atactic polystyrene-cyclohexane system at $308.2 \mathrm{~K}$.

experimental findings that $\left(\partial A_{2} / \partial T\right)_{\theta}$ is practically independent of $\bar{M}_{w}$ can be accepted by inspecting Fig. 2 because they employed PS samples in the $\bar{M}_{w}$ range of $1-18.1 \times 10^{4}$, in which all literature data points of $\psi_{0}$ (and $\kappa_{0}$ ) can be considered as nearly constant. It is probably true to say that at the limit of $\bar{M}_{w}\left(\right.$ or $\left.\bar{M}_{n}\right) \rightarrow \infty$ both $\psi_{0}$ and $\kappa_{0}$ at $\theta$ approach zero concurrently (see eq 28 ).

Although all the methods proposed so far for analyzing the critical solution points assume that $\chi$-parameter is independent of the polymer molecular weight, this assumption is not often realistic. For example, from analysis on the molecular weight distribution of polymers partitioning in a polymer-rich phase and a polymer-lean phase, both in equilibrium, Kamide et al. ${ }^{27,28}$ determined for the PS- $\mathrm{CH}$ system the partition coefficient $\sigma$, concluding the $\chi$-parameter to be slightly dependent of the polymer molecular weight and $\chi_{0}$ in eq 20 can be expressed semi-empirically over the limited $X_{w}$, range by

$$
\chi_{0}=\chi_{00}\left\{1+\left(k_{\chi} / X_{n}\right)\right\}
$$

with

$$
k_{\chi} \simeq k_{0}\{1-(\theta / T)\}
$$

$k_{\chi}$ was shown to decrease noticeably with increase in $T$, vanishing at $\theta$. The absolute magnitude of $k_{0}$ has unavoidably large experimental uncertainty.

The spinodal equation and equation of 
neutral equilibrium condition were derived by Kamide et al., ${ }^{16}$ for the case where $\chi$ and $\chi_{0}$ were given by eq 20 and 23, respectively. The results are:

$$
\begin{gathered}
1 /\left(X_{w} v_{\mathrm{p}}\right)+1 /\left(1-v_{\mathrm{p}}\right) \\
-\chi_{00}\left\{1+k_{\chi}\left(1+1 / X_{n}-X_{w} / X_{n}\right)\right\} \\
\times\left\{2+\sum_{i=1} p_{i}(i+2) v_{\mathrm{p}}{ }^{i}\right\}=0 \\
1 /\left(1-v_{\mathrm{p}}\right)^{2}-X_{z} /\left(X_{w} v_{\mathrm{p}}\right)^{2} \\
-\chi_{00}\left\{1+k_{\chi}\left(1+1 / X_{n}-X_{w} / X_{n}\right)\right\} \\
\times\left\{\sum_{i=1} p_{i} i(i+2) v_{\mathrm{p}}{ }^{i-1}\right\}=0
\end{gathered}
$$

By putting $k_{\chi}, X_{n}$, and $X_{w}$ into eq $18^{\prime}$ and 19' we obtain $v_{\mathrm{p}}{ }^{\mathrm{c}}$ and $\chi_{00}^{\mathrm{c}}\left(\chi_{00}\right.$ at the critical point $)$, together with $p_{1}$ and $p_{2}$, for a given polymer in solvent.

$\chi_{0}$ is related to $\psi_{0}$ and $\kappa_{0}$ through the relation

$$
1 / 2-\chi_{0}=\psi_{0}-\kappa_{0}
$$

and $\psi_{0}$ and $\kappa_{0}$, corresponding to $\chi_{0}$, given by eq 23 , can be approximately represented by eq 26 and 27 , respectively.

$$
\begin{aligned}
& \psi_{0}=\psi_{00}\left(1+k_{\psi} / X_{n}\right) \\
& \kappa_{0}=\kappa_{00}\left(1+k_{\kappa} / X_{n}\right)
\end{aligned}
$$

Figure 2 provides experimental support to confirm the validity of eq 26 and 27 over the range of $\bar{M}_{w}$, below $10^{6}$, but eq 26 and 27 are shown to be not fully satisfactory when a very large molecular weight range is considered. $\kappa_{00}$ is an apparent value extrapolated to $\bar{M}_{w^{\prime}}$ (or $\left.\bar{M}_{n}\right)=\infty$ from the data points in the limited $\bar{M}_{n^{\prime}}$ range. So $\kappa_{00}$ is similar to the values at the intercept of lines a, b, and c in Figure 2. $\kappa_{00}$ does not necessarily mean a true $\kappa_{0}$ value at infinite molecular weight.

Equation 21 can be modified into

$$
1 / T_{\mathrm{c}}=\chi_{00}^{\mathrm{c}} /\left(\theta \psi_{00}\right)-\left(1 /\left(\theta \psi_{00}\right)\right)\left(1 / 2-\psi_{00}\right)
$$

Then, a graph of $1 / T_{c}$ as a function of $\chi_{00}^{c}$

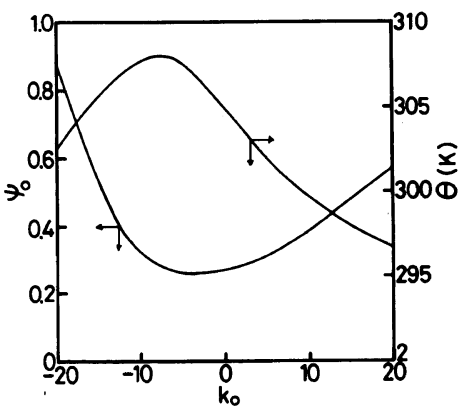

Figure 4. Effect of the molecular weight dependence coefficient $k_{0}$ (eq 24) on Flory theta temperature $\theta$ and Flory entropy parameter at infinite dilution $\psi_{0}$, both evaluated by the Kamide-Matsuda method (eq 18 and 19) from the critical solution points data, for the atactic polystyrene-cyclohexane system.

enables $\theta$ and $\psi_{00}$ to be evaluated from the intercept and the slope simultaneously.

In order to clarify the effect of the molecular weight dependence of $\chi$ on $\psi_{00}$ we calculated $\theta$ and $\psi_{0}$ using eq $21^{\prime}$ assuming $k_{0}$ for literature data. ${ }^{8,12,13}$ The results are shown in Figure 4. At $k_{\chi}=0, \psi_{00}=0.27$ and $\theta=305.1 \mathrm{~K}$ are obtained. As noted previously, ${ }^{29} \theta$ values estimated by the Koningsveld et al. method and by the Kamide-Matsuda method are by a few degrees lower than the temperature at which $A_{2}$ vanishes. Provided that underestimation of $\theta$ by these two methods is entirely due to undue neglect of the molecular weight dependence of the $\chi$-parameter and eq 23 and 24 hold their validity over an whole $X_{w}$, range including infinite molecular weight, $\psi_{00}$ (and accordingly $\kappa_{00}$ at $\theta$ ) and $k_{0}$ corresponding to the true $\theta$ are considered to be the most probable values. From Figure 4, we obtain $\psi_{00}=0.26 \pm 0.01$ and $k_{0}=-4.6$, corresponding to $\theta=307.5 \pm 0.01 \mathrm{~K}$ for PS-CH system. It is noteworthy that the $\psi_{00}$ (accordingly, $\kappa_{00}$ at $\theta$ ) value is equivalent to that extrapolated from the data points in the range of $\bar{M}_{w}=3.45 \times$ $10^{4}-270.0 \times 10^{4}$. The former value should be compared with 0.27 , which was derived assuming that $\chi$-parameter is molecular weight independent.

Figure 2 indicates that except for the points 


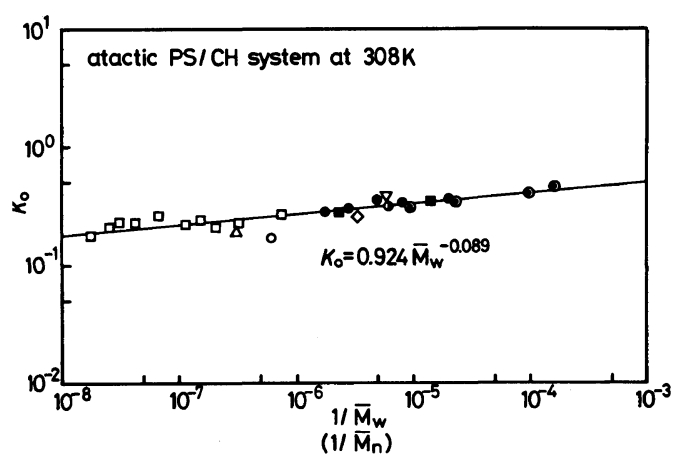

Figure 5. Log-log plot of Flory enthalpy parameter at infinite dilution $\kappa_{0}$, evaluated by the temperature dependence of the chemical potential and second virial coefficient $A_{2}$ in the vicinity of the theta temperature and by calorimetry versus the reciprocal weight- (or number-) average molecular weight $\bar{M}_{w}\left(\bar{M}_{n}\right)$ for the atactic polystyrene-cyclohexane system: $\mathbf{O}, \mathrm{Krigbaum}^{3}$ (membrane osmometry (MO)); $\square$, KrigbaumGeymer $^{10}$ (MO); O, Scholte ${ }^{11}$ (ultracentrifuge); $\triangle$, Krigbaum-Carpenter $^{6}$ (light scattering (LS)); $\nabla$, Schulz-Baumann ${ }^{18}$ (LS); $\diamond$, Kotera et al. ${ }^{19}$ (LS); $\bigcirc$, Outer et al. ${ }^{17}$ (LS); $\square$, Miyaki ${ }^{21}$ (LS); $\bigcirc$, Fujihara ${ }^{25}$ (calorimetry). Solid line, the equation $\kappa_{0}=0.92 \bar{M}_{w(n)}^{-0.089}$.

by Schulz and Baumann ${ }^{18}$ and by Krigbaum ${ }^{3}$ (sample code $\mathrm{H}-2-8$ ), all the data points estimated by numerous researchers using various methods seem to be represented by a single master curve, which very gradually decreases with $\bar{M}_{w}$ above $\bar{M}_{w}>10^{6}$. In order to obtain a better understanding of the molecular weight dependence of $\kappa_{0}$, a log-log plot of $\kappa_{0}$ against $\bar{M}_{w}{ }^{-1}$ or $\bar{M}_{n}{ }^{-1}$ for atactic PS-CH system at $307.2 \mathrm{~K}$ is shown in Figure 5. All available data points yielded a straight line given by

$$
\kappa_{0}\left(\equiv \psi_{0}\right)=0.924 \bar{M}_{w}\left(\text { or } \bar{M}_{n}\right)^{-0.089}
$$

Equation 28 is valid over the entire experimentally accessible molecular weight range from $6.2 \times 10^{3}$ to $5.680 \times 10^{7}$ and of course eq 28 has much wider applicability than eq 27 . Equation 28 is represented by the full line in Figure 2. Therefore, we can conclude that the most probable $\kappa_{0}$ value is, in a strict sense, dependent on $\bar{M}_{w}$ (or $\bar{M}_{n}$ ), irrespective of the method employed and that if eq 28 can be expanded its applicability to $\bar{M}_{w}=\infty \kappa_{0}$ at
Table III. The Flory $\theta$ temperature and entropy parameter $\kappa_{0}$ at infinite dilution for the upper critical solution point of the atactic polystyrene-trans-decalin system

\begin{tabular}{llll}
\hline Method & $\theta / \mathrm{K}$ & $\kappa_{0}$ at $\theta$ \\
\hline Critical point & & \\
& Shultz-Flory & 293.7 & 0.95 \\
& Kamide-Matsuda & 292.7 & 0.33 \\
Second virial coefficient & & \\
$\quad$ Light scattering & 296.8 & 0.32 \\
\hline
\end{tabular}

infinite molecular weight may be zero. This is an experimental indication that both the randomness in the mixing of a polymer and solvent and the spacial homogeneity of the polymer segment density in solution are expected to be realized in dilute solutions of polymer with infinitely large molecular weight (i.e., $\Delta S_{0}=\Delta S_{0}{ }^{\text {comb }}$ ). The fact that methods (1) - (4) give essentially identical $\kappa_{0}$ value within \pm 0.02 for a given PS sample in $\mathbf{C H}$, strongly supports the validity of the modified Flory-Huggins theory.

For the atactic PS-trans-decalin system, upper critical solution points and temperature dependence of the second virial coefficient were reported in literature. Nakata et al. ${ }^{30}$ determined $v_{\mathrm{p}}^{\mathrm{c}}$ and $T_{\mathrm{c}}$ for four monodisperse PS samples $\left(\bar{M}_{x^{\prime}}=3.7-270 \times 10^{4}\right)$ in transdecalin. In the previous paper, ${ }^{29}$ we estimated $\theta$ and $\psi_{0}$ from the above data according to the Shultz-Flory, Kamide-Matsuda methods. The results are shown in Table III, where $\kappa_{0}$ values at $\theta$ calculated from $\psi_{0}$ are shown.

Inagaki and his coworkers ${ }^{31}$ measured the light scattering second virial coefficient $A_{2}$ of four well-fractionated PS samples $\left(\bar{M}_{w}=\right.$ $\left.14.3-112 \times 10^{4}\right)$ in trans-decalin over temperatures ranging 291.2 to $349.2 \mathrm{~K}$ and evaluated $\theta$ and $\left(\partial A_{2} / \partial T\right)_{\theta}$ (Table III of ref 22), from which we calculated $\psi_{0}$ and $\kappa_{0}$ at $\theta$ assuming $\left(V_{1} / \bar{v}^{2}\right)=183.82 \mathrm{~g}^{2} \mathrm{~cm}^{3} \mathrm{~mol}^{-1}$, regardless of temperature. The results are summarized in Table IV. From the plot of $\kappa_{0} v s$. $1 / \bar{M}_{w}$ (Figure 6), $\kappa_{0}$ at infinite molecular 
Table IV. Evaluation of Flory $\kappa_{0}$ parameter from the temperature dependence of the second virial coefficient $A_{2}$ by light scattering for atactic polystyrene-trans-decalin system

\begin{tabular}{|c|c|c|c|c|c|c|c|}
\hline \multirow{2}{*}{ Method } & \multirow{2}{*}{ Reporter (Year) } & \multirow{2}{*}{$\begin{array}{l}\text { Sample } \\
\text { code }\end{array}$} & \multirow{2}{*}{$\frac{\bar{M}_{w}}{\times 10^{-4}}$} & \multirow{2}{*}{$\frac{\text { Temp }}{\mathrm{K}}$} & \multirow{2}{*}{$\frac{\left(\partial A_{2} / \partial T\right)_{\theta}}{\times 10^{6} \mathrm{~cm}^{3} \mathrm{molg}^{-1} \mathrm{~K}}$} & \multirow{2}{*}{$\frac{\theta}{\mathbf{K}}$} & \multirow{2}{*}{$\begin{array}{c}\kappa_{0} \\
\text { at } \Theta^{\text {a }}\end{array}$} \\
\hline & & & & & & & \\
\hline \multirow{4}{*}{$\begin{array}{l}\text { Light } \\
\text { scattering }\end{array}$} & \multirow{4}{*}{$\begin{array}{l}\text { Inagaki et al. } \\
\quad(1966)\end{array}$} & G-2 & 14.3 & $291.2-343.2$ & 1.43 & 296.8 & 0.78 \\
\hline & & $\mathrm{J}-4$ & 22.2 & $295.5-302.1$ & 1.14 & 296.8 & 0.62 \\
\hline & & $\mathrm{J}-10$ & 51.3 & $295.2-300.1$ & 1.11 & 296.7 & 0.61 \\
\hline & & P-4 & 112 & $295.2-302.8$ & 0.69 & 296.8 & 0.38 \\
\hline
\end{tabular}

a $\left(V_{1} / \bar{v}^{2}\right)=183.82$

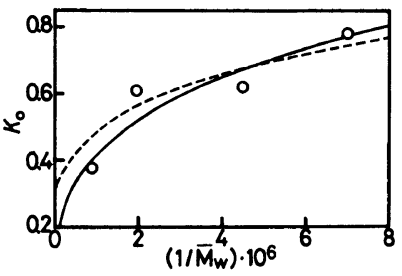

Figure 6. The molecular weight dependence of Flory enthalpy parameter at infinite dilution $\kappa_{0}$, evaluated by Inagaki et al. ${ }^{31}$ from the temperature dependence of the second virial coefficient $A_{2}$, for the atactic polystyrenetrans-decalin system.

weight was found to be 0.32 (Table III). $\kappa_{0}$ can also be experimentally represented by

$$
\kappa_{0}=31.5 \bar{M}_{w}{ }^{-0.312}
$$

The $\kappa_{0}$ value from the critical point by Kamide-Matsuda treatment $(0.33)$ for the atactic PS-trans-decalin system agrees fairly well with that from $\left(\partial A_{2} / \partial T\right)_{\theta}(0.32)$ because the molecular weight ranges of these two sets of data overlap fairly well.

Summarizing, this article clarified the reliability of $\kappa_{0}$ in Flory theta solvent experimentally estimated and a molecular weight dependence of $\kappa_{0}$ for typical non-polar polymernon-polar solvent systems. Then, we come to the conclusion that the modified FloryHuggins theory, derived by introducing the molecular weight- and the concentrationdependences of the $\chi$ parameter into the original theory, can be regarded as valid and sufficiently accurate to express any thermodynamic property of a polymer solution.

\section{REFERENCES}

1. See, for example, P. J. Flory, "Principle of Polymer Chemistry," Cornell Univ. Press, Ithaca, New York, 1953.

2. See, for example, A. Kagemoto, S. Murakami, and R. Fujishiro, Bull. Chem. Soc. Jpn., 39, 15 (1966).

3. W. R. Krigbaum, J. Am. Chem. Soc., 76, 3785 (1954).

4. A. R. Shultz and P. J. Flory, J. Am. Chem. Soc., 74, 4760 (1952).

5. T. G. Fox, Jr. and P. J. Flory, J. Am. Chem. Soc., 73, 1915 (1951).

6. W. R. Krigbaum and D. K. Carpenter, J. Phys. Chem., 59, 1166 (1955).

7. W. H. Stockmayer, J. Chem. Phys., 17, 588 (1949).

8. R. Konigsveld, L. A. Kleintjens, and A. R. Shultz, J. Polym. Sci., A-2, 8, 1261 (1970).

9. K. Kamide and S. Matsuda, Polym. J., 16, 825 (1984).

10. W. R. Krigbaum and D. O. Geymer, J. Am. Chem. Soc., 81, 1859 (1959).

11. Th. G. Scholte, J. Polym. Sci., A-2, 8, 841 (1970).

12. N. Kuwahara, N. Nakata, and M. Kaneko, Polymer, 14, 415 (1973).

13. S. Saeki, N. Kuwahara, S. Konno, and M. Kaneko, Macromolecules, 6, 246 (1973).

14. K. Kamide, Y. Miyazaki, and T. Abe, Makromol. Chem., 177, 485 (1976).

15. K. Kamide, Y. Miyazaki, and T. Abe, Polym. J., 9, 395 (1977).

16. K. Kamide, S. Matsuda, T. Dobashi, and $\mathbf{M}$. Kaneko, Polym. J., 16, 839 (1984).

17. P. Outer, C. I. Carr, and B. H. Zimm, J. Chem. Phys., 18, 830 (1950).

18. G. V. Schulz and H. Baumann, Makromol. Chem., 60, 120 (1963). 
19. A. Kotera, T. Saito, and N. Fujisaki, Rep. Progr. Polym. Phys. Jpn., 6, 9 (1963).

20. Y. Miyaki and H. Fujita, Macromolecules, 14, 742 (1981).

21. Y. Miyaki, PhD. Dissertation, 1981, Osaka Univ.

22. Z. Tong, S. Ohashi, Y. Einaga, and H. Fujita, Polym. J., 15, 835 (1983).

23. K. Amaya and R. Fujishiro, Bull. Chem. Soc., Jpn., 31, 19 (1958).

24. G. V. Schulz and A. Horbach, Z. Phys. Chem. Neue Folge, 22, 377 (1959).

25. I. Fujihara, PhD. Dissertation, 1979, Osaka City Univ.
26. H. J. Cantow, Z. Phys. Chem. Neue Folge, 7, 58 (1956).

27. K. Kamide and Y. Miyazaki, Polym. J., 13, 325 (1981).

28. K. Kamide, T. Abe, and Y. Miyazaki, Polym. J., 14, 355 (1982).

29. K. Kamide, S. Matsuda, and M. Saito, Polym. J., 17, 1013 (1985).

30. M. Nakata, S. Higashida, N. Kuwahara, S. Saeki, and M. Kaneko, J. Chem. Phys., 64, 1022 (1976).

31. H. Inagaki, H. Suzuki, M. Fujii, and T. Matsuo, J. Phys. Chem., 70, 1718 (1966). 